\title{
Investigation of the dp-non-mesonic breakup reaction at $300-500 \mathrm{MeV}$ at Nuclotron
}

\section{S.M. Piyadin*, M.Janek ${ }^{1,2}$, G.Tarjanyiova ${ }^{2}$, Yu.V. Gurchin ${ }^{1}$, A.Yu. Isupov ${ }^{1}$,} J.-T. Karachuk ${ }^{1,3}$, A.N. Khrenov ${ }^{1}$, A.K. Kurilkin ${ }^{1}$, P.K. Kurilkin ${ }^{1}$, V.P. Ladygin ${ }^{1}$, A.N. Livanov ${ }^{1}$, G. Martinska ${ }^{4}$, S.G. Reznikov ${ }^{1}$, A.A. Terekhin ${ }^{1}$.

${ }^{1}$ Joint Institute for Nuclear Research, Dubna 141980, Russian Federation

${ }^{2}$ Physics Department, University of Žilina, Žilina 010 26, Slovak Republic

${ }^{3}$ Advanced Research Institute for Electrical Engineering, Bucharest 030138, Romania

${ }^{4}$ P.J.Šafarik University, Košice 041 80, Slovak Republic

E-mail: piyadinejinr.ru

The first results on the study of the $d p$-breakup reaction with $300-500 \mathrm{MeV}$ unpolarized deuteron beam at Internal Target Station at Nuclotron are discussed. Selection procedure of useful events for the $d p \rightarrow p p n$ reaction with the registration of two protons is shown.

XXII International Baldin Seminar on High Energy Physics Problems,

15-20 September 2014

JINR, Dubna, Russia

\footnotetext{
* Speaker.
} 


\section{Introduction}

The purpose of Deuteron Spin Structure experimental program is to obtain the information on

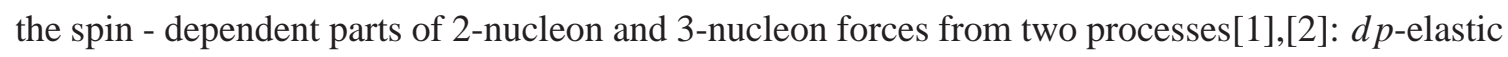
scattering in a wide energy range [3], [ [ [ 4 , [5], [6] and $d p$ non-mesonic breakup with two protons detection at energies $200-500 \mathrm{MeV}$ 77.

Properties of few-nucleon system at moderate energies are determined mainly by pairwise nucleon-nucleon interactions. Models of $N N$ forces describe the long range interaction part according to the meson-exchange, while the short range is based on phenomenology, adjusted by fitting a certain number of parameters to the $N N$ scattering data. Nowadays a new generation of the $N N$ potentials (AV-18[8], CD-Bonn[9], Nijmegen[10] etc.) was obtained. They reproduce data on the nucleon nucleon scattering up to $350 \mathrm{MeV}$ with very good accuracy. However, these modern $2 N$ forces fail to provide the experimental binding energies of few-nucleon systems. Moreover, the data on the $d p$ - elastic scattering and deuteron breakup are not described properly.

Precise predictions for observables in the $3 N$ system can be obtained via exact solutions of the $3 N$ Faddeev equations for any nucleon-nucleon $(N N)$ interaction, even with the inclusion of a $3 N F$ model [11]. Incorporation of the $3 N$ forces makes it possible to reproduce the binding energy of the three-nucleon bound systems and also data on unpolarized $d p$-interaction. Nevertheless, polarization data for the reactions with participation of three and more nucleons are not described even with inclusion of $3 N F$. Therefore, the obtaining of the additional polarization data in the $d p$ interaction in the wide energy range more is very desirable for the study of the spin structure of $2 N$ and $3 N$ forces [12]. To investigate the details of the dynamics of the $3 N$ system, in addition to elastic $N d$ scattering data, reliable deuteron breakup data sets, covering large regions of the available phase space, are needed.

The experimental data on the deuteron analyzing powers for $d p$-breakup for large phase space were obtained at $130 \mathrm{MeV}$ at KVI [13]. $A_{y}, A_{y y}$ and $A_{x x}$ analyzing powers in $d p$-breakup will be investigated at Internal Target Station at $200-500 \mathrm{MeV}$. The predictions for analyzing powers and differential cross section in the selected $d p$-breakup configurations at $200 \mathrm{MeV}$ were obtained in [14]. It was shown large sensitivity of these observables to the model of $3 N F$.

In this report the status of the experiment at internal target station (ITS) at Nuclotron preparation and first beam results are presented.

\section{The experimental setup}

The first stage of the DSS experimental program[2, 15, 16] is the beam energy scan of $d p$ - elastic scattering cross section at the deuteron energies from 400 up to $2000 \mathrm{MeV}$ and measurements of $d p$-non-mesonic breakup at 300, 400 and $500 \mathrm{MeV}$ in different kinematic configurations. These measurements will be performed using ITS[17] with new control and data acquisition system [18].

The $d p$ breakup reaction will be investigated using $\Delta E-E$ techniques for the detection of protons. 8 detectors of this kind are planned to use in the experiment. The details of the $\Delta E-E$ detector construction are presented in [7], [19].

Each detector consists of two scintillators $\Delta E$ and $E$. The first scintillator has the cylindrical form with the height $10 \mathrm{~mm}$ and the diameter $80 \mathrm{~mm}$. Two PMTs- 85 view through given scintillator 
and they are located the friend opposite to the friend. Two planes have been made on the scintillator to increase the area of the optical contact between the scintillator and photocatode of the PMT-85. These planes have been polished. $\Delta E$ scintillator is covered by a white paper. Digital dividers of the high voltage are used for PMT-85.

$E$ scintillator also has the cylindrical form with height $200 \mathrm{~mm}$ and diameter $100 \mathrm{~mm}$. PMT63 view through this scintillator. Given PMT has been chosen because of the suitable size of the cathode $(100 \mathrm{~mm})$ with both good time and amplitude properties. $E$ scintillator has been wrapped by a white paper. The part which is located to $\Delta E$ scintillator has been covered by a black paper. It is made to exclude possibility of hit the light from one plastic scintillator to another. The calibration for $\Delta E-E$ counters were performed with cosmic muons and $p p$-quasi elastic scattering.

\section{The simulation results}

The simulation results on the energy losses of the protons which passes through the scintillators are presented in Fig. 1. In (b) figure the energy losses of the proton in thin scintillator are plotted versus ones of the thick scintillator. The events were selected in the region of approximate linear dependence of the energy losses in the scintillator versus the incident proton energy [20]. Therefore the following conditions on the $\Delta E$ and $E$ information were imposed:

$$
5 \mathrm{MeV}<\Delta E<35 \mathrm{MeV} \text { and } 0 \mathrm{MeV}<E<180 \mathrm{MeV} \text {. }
$$
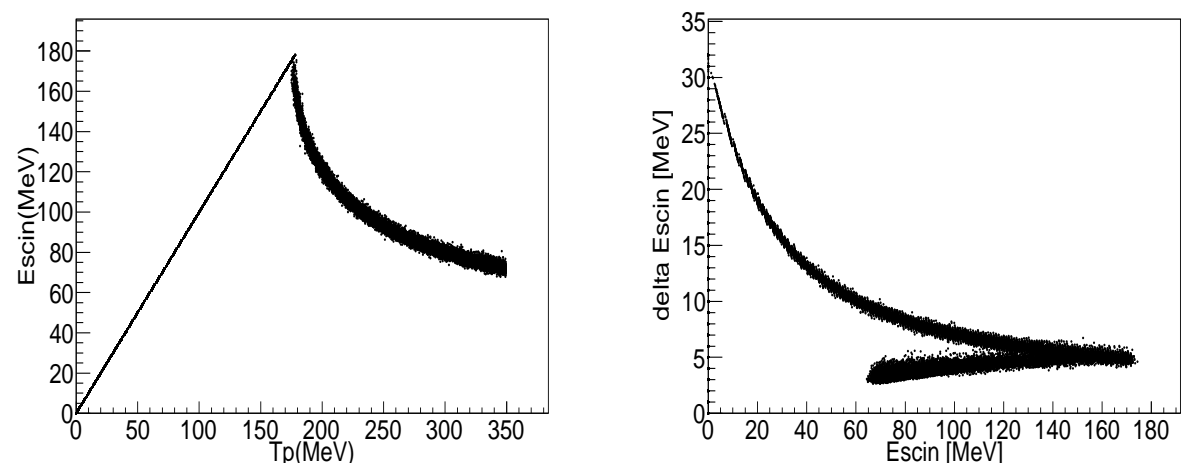

Figure 1: (a) Simulation results of the energy losses of the protons passed through $\Delta E$ and $E$ scintillators when the length of a scintillator is $21 \mathrm{~cm}(\Delta E+E)$ (b) Simulation results of the energy losses of the protons passed through $\Delta E$ and $E$ scintillators in thin scintillator versus thick.

\section{Results obtained at Nuclotron.}

The missing mass has been calculated using the kinematic formulas (Fig. 2). Histogram was obtained for the kinematical configuration $\Theta_{1}=25^{\circ}, \Theta_{2}=43.6^{\circ}, \Phi_{12}=178.5^{\circ}$ and deuteron energy of $400 \mathrm{MeV}$. One can see clearly the allocated the $d p$-breakup reaction and $d p$-elastic scattering. Useful events were obtained with the cut on missing mass: less than $950 \mathrm{MeV}$ for the configuration register $d p$-elastic scattering and $d p$-breakup are detected; $940 \mathrm{MeV} \pm 10 \mathrm{MeV}$ for the configuration when only $d p$-breakup events are registered. 


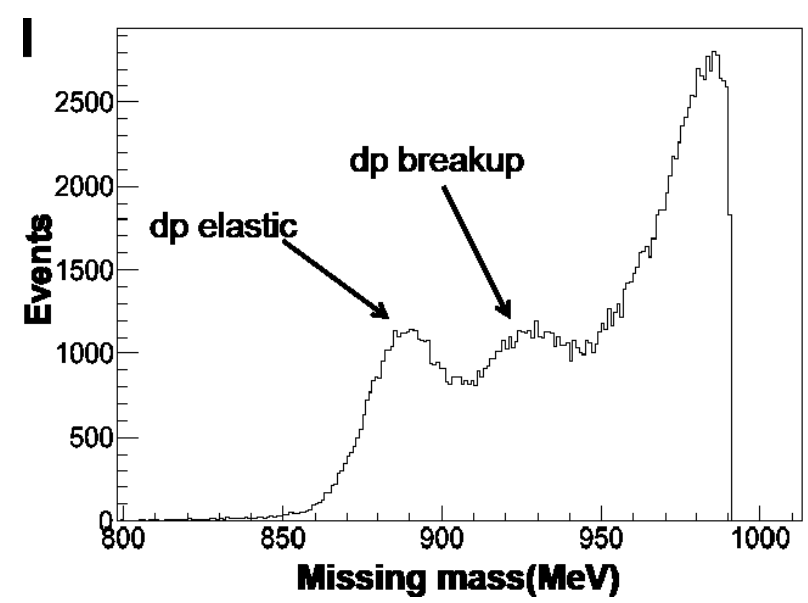

Figure 2: The missing mass for the kinematical configuration $\Theta_{1}=25^{\circ}, \Theta_{2}=43.6^{\circ}, \Phi_{12}=178.5^{\circ}$.

Correlations of the detected proton energies with the cut on the neutron missing mass obtained at $300 \mathrm{MeV}, 400 \mathrm{MeV}$ and $500 \mathrm{MeV}$ on $\mathrm{CH}_{2}$ target are presented in the left, middle and right panels of Fig. B.
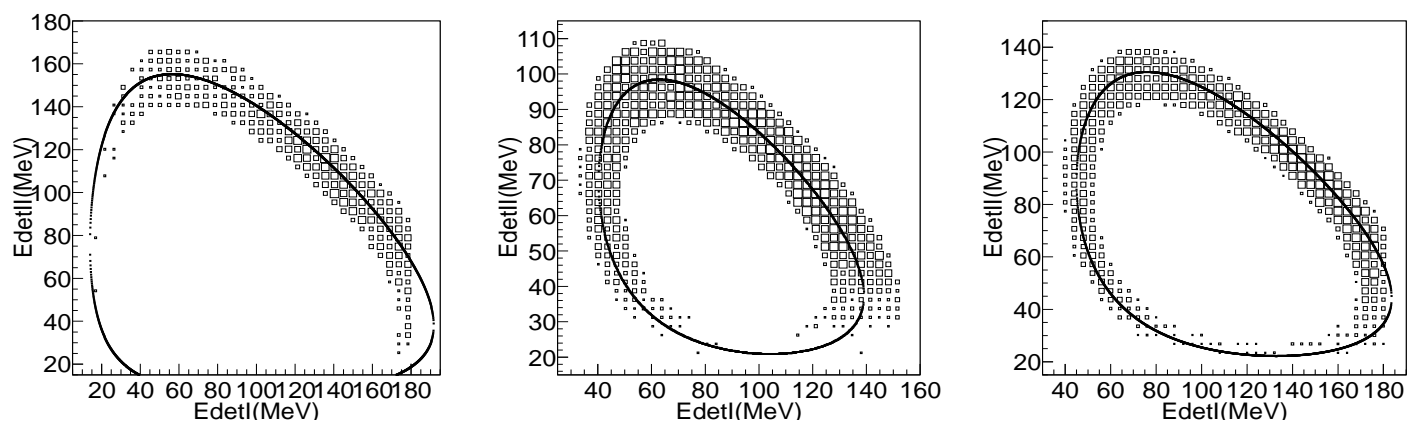

Figure 3: The missing mass for the kinematical configuration $\Theta_{1}=25^{\circ}, \Theta_{2}=43.6^{\circ}, \Phi_{12}=178.5^{\circ}$.

The spectra correspond to the detected protons kinematical configuration $\Theta_{1}=25^{\circ}, \Theta_{2}=43.6^{\circ}$, $\Phi_{12}=178.5^{\circ}$. Black curve is the kinematic locus for deuteron breakup reaction at $300-500 \mathrm{MeV}$ energy of deuteron for different angle configuration. The correlation of the proton energies were ploted with the cut on mass of neutron $( \pm 10 \mathrm{MeV})$.

\section{Conclusion}

The preliminary results for $d p \rightarrow p p n$ reaction at 300-500 MeV for different geometry at Internal Target Station at Nuclotron are obtained. The procedure of selection of events relating to the dp-breakup reaction is established. The setup for the studies of deuteron non-mesonic breakup reaction was put into operation.

The future plans are to continue to process the obtained data and to estimate a contribution of background events to these results for the study of dp-breakup reaction. We are going to take the 
data on dp - breakup using with polarized deuteron beams at Nuclotron. New source will provide up to $2 * 10^{10} \mathrm{ppp}$ and higher values of polarization than POLARIS. Part of the IUCF source is used for the construction. The putting into operation of new PIS is planned in 2016.

The work has been supported in part by RFBR grant 13-02-00101a.

\section{References}

[1] V.P. Ladygin et al., Short-range correlations studies in collisions of polarized nuclei at Nuclotron-M, EPJ Web Conferences, 3, (2010) 04004

[2] V.P. Ladygin et al., Few-body Studies at Nuclotron-JINR, Few Body Syst. 55 (2014) 709.

[3] P.K.Kurilkin et al., Measurement of the vector and tensor analyzing powers in dp elastic scattering at the energy of 880 MeV, Eur.Phys.J. Special Topics 162 (2008) 137.

[4] P.K. Kurilkin et al., Investigation of the angular dependence of the analyzing powers in the deuteron-proton elastic scattering at the nuclotron, Phys.Part. and Nucl.Lett. 8 (2011) 1081.

[5] A.Terekhin et al.Study of dp-elastic scattering at energies 650, 750 and $1000 \mathrm{MeV/nucleon}$, Nucl.Phys.Proc.Suppl. 245 (2013) 185.

[6] Yu.Gurchin et al. The differential cross-section on dp-elastic scattering at 400-880 MeV obtained at Nuclotron, Nucl.Phys.Proc.Suppl. 245 (2013) 271.

[7] S.M.Piyadin et al., ÎŤE-E detector for proton registration in nonmesonic deuteron breakup at the nuclotron internal target, Phys.Part. and Nucl.Lett. 8 (2011) 107.

[8] R.B. Wiringa, V.G.J. Stoks, and R. Schiavilla., AN ACCURATE NUCLEON-NUCLEON POTENTIAL WITH CHARGE INDEPENDENCE BREAKING, Rhys. Rev., C51, (1995) 38.

[9] R. Machleidt, THE HIGH PRECISION, CHARGE DEPENDENT BONN NUCLEON-NUCLEON POTENTIAL (CD-BONN), Phys.Rev., C63, (2001) 024001.

[10] V.G.J. Stoks, R.A.M. Klomp, C.P.F. Terheggen, and J.J. de Swart., CONSTRUCTION OF HIGH QUALITY N N POTENTIAL MODELS, Rhys. Rev. C49, (1994) 2950.

[11] W. Glockle, H. Witala, D. Huber, H. Kamada, and J. Golak, The three-nucleon continuum: achievements, challenges and applications, Phys. Rep. 274, (1996) 107.

[12] T. Uesaka, V.P. Ladygin et al., Proposal on the measurements of d-p elastic scattering analyzing powers at 0.3-2.0 GeV at internal target station of the nuclotron, Part.Nucl. and Nucl. Lett. 3 (2006), 305.

[13] St. Kistryn et al., Systematic study of three-nucleon force effects in the cross section of the deuteron-proton breakup at 130-MeV, Phys.Rev., C72, (2005), 044006.

[14] J. Kuros-Zolnierczuk et al., Three-nucleon force effects in nucleon induced deuteron breakup. I. Predictions of current models, Phys.Rev., C66, (2002), 024003.

[15] V.P. Ladygin et al., Recent results with polarized deuterons and polarimetry at Nuclotron-NICA, J.Phys.Conf.Ser. 295 (2011) 012131.

[16] V.P. Ladygin et al., Spin physics in few body systems at Nuclotron, Phys.Part.Nucl. 45 (2014) 327.

[17] A.I. Malakhov et al., Potentialities of the internal target station at the Nuclotron, Nucl.Instrum.Meth. in Phys.Res. A440 (2000) 320. 
[18] A.Yu. Isupov, V.A. Krasnov, V.P. Ladygin, S.M. Piyadin, S.G. Reznikov, The Nuclotron internal target control and data acquisition system, Nucl.Instrum.Meth. in Phys.Res. A698 (2013) 127.

[19] S.M.Piyadin et al., Experiments on the study of the deuteron-proton interactions at intermediate energies at Internal Target at Nuclotron, Phys.Part. and Nucl.Lett. 8 (2011) 1084.

[20] M.Janek et al., Experimental and simulated dp breakup reaction data at 300, 400 and $500 \mathrm{MeV}, \mathrm{EPJ}$ Web Conf. 81 (2014) 06005. 